\title{
TEM Study of the Structural Properties of Nanowires Based on Cd, Zn, Te grown by MBE on Silicon Substrates
}

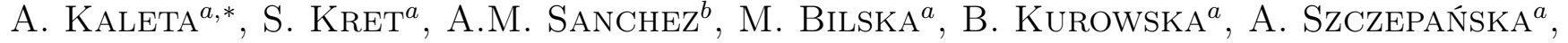 \\ J. PŁACHTA ${ }^{a}, \mathrm{P}$. WOJNAR ${ }^{a}$ AND T. WOJCIECHOWSKI ${ }^{a}$ \\ ${ }^{a}$ Institute of Physics, Polish Academy of Sciences, Aleja Lotnikow 32/46, PL-02668 Warsaw, Poland \\ ${ }^{b}$ Department of Physics, University of Warwick, Coventry CV4 7AL, UK
}

\begin{abstract}
In this work we report on the atomic structures, elemental distribution, defects and dislocations of three types of semiconductor nanowires: ZnTe, CdTe, and complex ZnTe/(Cd,Zn)Te core/shell hetero-nanowires grown by a molecular beam epitaxy on (111) Si substrate using a vapor-liquid-solid mechanism. The structural properties and the chemical gradients were measured by transmission electron microscopy methods. The nanowires reveal mainly sphalerite structure, however wurtzite nanowires were also observed.
\end{abstract}

DOI: 10.12693/APhysPolA.131.1399

PACS/topics: 81.05.Dz, 81.07.-b, 61.46.-w, 62.23.Hj, 81.07.Gf, 61.46.Km, 78.67.Uh, 07.10.Pz, 68.35.Gy, 68.37.Lp, 68.37.Og, 68.37.Ma

\section{Introduction}

Nowadays the nanostructures attract great interest as they reveal new, different properties than their bulk equivalents. The nanowires are $1 \mathrm{D}$ nanostructures having the length hundred of times bigger than their diameter which implies the high surface to volume ratio characteristic. Therefore, the nanowires (NWs) are becoming extremely promising materials for future nanoscale electronics, optics, and photonics. One of the key reasons is the eradication of the lattice/thermal coefficient constraint with the substrate. Thus, the substrate does not govern the NW crystal structure. Devices based on NWs, such as a single electron transistor [1], photodetectors [2], lasers [3] and photovoltaic cells [4] have already been demonstrated. However, there is still a challenge for a development in producing repeatable, good-quality NWs for the efficient application in the technology.

The main building materials for such NWs are semiconductors of direct band gaps ensuring good optical properties from III-V groups: GaAs, GaN, InGaN, AlGaN, InAs, InP, InN, and II-VI groups: ZnO, ZnSe, CdSe, $\mathrm{CdS}$. Interestingly, the semiconductors made of Te anions also seem to be the appropriate materials for the optoelectronic devices as they have the direct band gaps covering the visible spectrum energies [5] from $1.5 \mathrm{eV}$ (CdTe) to $2.3 \mathrm{eV}(\mathrm{ZnTe})$ and even $3.4 \mathrm{eV}(\mathrm{MgTe})$, however they do not reveal the near band edge (NBE) emission $[6,7]$.

Lately, the successful activation of the NBE emission $(2.33 \mathrm{eV})$ for the ZnTe NW has been reported by Wojnar et al. [8] as a result of the surface states passivation $[9,10]$ achieved by coating the NW with a higher band gap shell (ZnMgTe). The use of the Si substrates in the work [8] was the first attempt to integrate the II-VI NWs with the well-established Si technology

*corresponding author; e-mail: kaleta@ifpan.edu.pl as so far the NWs were grown on the GaAs substrates in orientations (001), (110), (111)B [8]. Additionally, the substantial differences between the $\mathrm{GaAs}(111) \mathrm{B}$ and the $\mathrm{Si}(111)$ substrates used for $\mathrm{ZnTe} / \mathrm{ZnMgTe}$ core/shell NWs grown by the molecular beam epitaxy (MBE) via vapor-liquid-solid (VLS) mechanism [11] with gold droplets as the catalyst were discussed in [9]. (111)NWs grown on GaAs (111)B were perpendicular to the substrate surface, whereas the (111)NWs grown on the Si(111) had a random growth orientation in reference to the $\mathrm{Si}$ substrate. Moreover, the NBE emission was at least one order of magnitude higher for the NWs grown on the $\mathrm{Si}(111)$. It was possible that the Ga precipitations from $\mathrm{Au} / \mathrm{Ga}$ eutectic droplet might have been incorporated into the NWs structure which resulted in deep level defect states trapping the band carriers and reducing the NBE emission. Nevertheless, the exact reason of the low emission is not completely understood.

A subsequent concept which has been tried out was making an optically active axial nano-insertion of a different material in the NW structure $[12,13]$ - i.e. the quantum dots in the NWs. The first trial was to grow a $(\mathrm{Cd}, \mathrm{Zn}) \mathrm{Te}$ segment on the ZnTe NW. The proper incorporation of the $\mathrm{Cd}$ atoms into the ZnTe lattice needs a temperature reduction to overcome the additional thermodynamic barrier related to the difference in lattice constants between ZnTe and CdTe (Table I). The temperature chosen for the $(\mathrm{Cd}, \mathrm{Zn}) \mathrm{Te}$ segment needs to be higher than the $\mathrm{Au} / \mathrm{Si}$ melting temperature otherwise the top golden droplet "freezes" and the VLS mechanism stops.

In this work we investigate three types of II-VI semiconductor NWs grown by the MBE via the VLS mechanism with gold catalyst on (111)Si substrate: ZnTe NWs, CdTe NWs and the corresponding ZnTe/(Cd,Zn)Te core/shell heterostructure NWs. Both the ZnTe and the CdTe compounds have the zinc blende (sphalerite) structure (cubic in the equilibrium conditions) with a direct band gap $E_{g}=2.4 \mathrm{eV}$ and $E_{g}=1.5 \mathrm{eV}$, respectively. Although their symmetry is compatible with the Si sub- 
strate, the growth of II-VI semiconductor compounds on the $\mathrm{Si}$ is difficult caused by the fundamental problems such as a high lattice mismatch (see Table I).

Transmission electron microscopy (TEM) analyses have been carried out to correlate the atomic structure/defect density with the II-VI NWs physical properties (e.g. luminescence). In the case of $\mathrm{ZnTe} /(\mathrm{Cd}, \mathrm{Zn}) \mathrm{Te}$ NWs, the TEM investigations can give a clear answer whether during the growth of the $(\mathrm{Cd}, \mathrm{Zn}) \mathrm{Te}$ segment axial growth by the VLS mechanism was maintained.

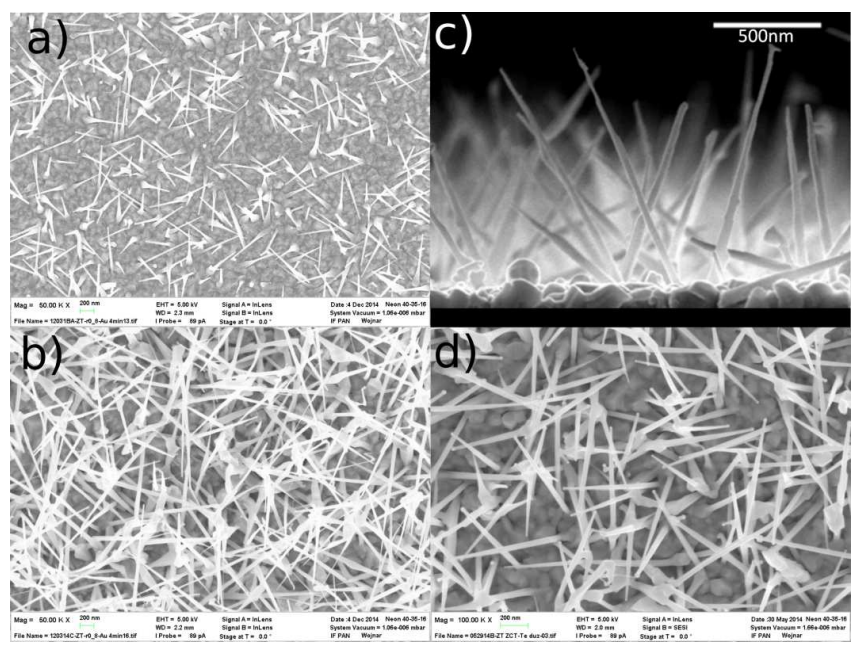

Fig. 1. SEM images of NWs grown on (111) Si substrate and $2 \mathrm{D}$ island between the surface and the NWs (a) A ZnTe NWs, (b) B ZnTe NWs, (c) CdTe NWs, (d) $\mathrm{ZnTe} /(\mathrm{Cd}, \mathrm{Zn}) \mathrm{Te}$ core/shell NWs.

TABLE I

Band gaps and lattice parameters for the selected II-V semiconductor compounds and the silicon substrate. Misfits with the substrate and the different core/shell heterostructures are also presented.

\begin{tabular}{|c|c|c|c|c|c|}
\hline 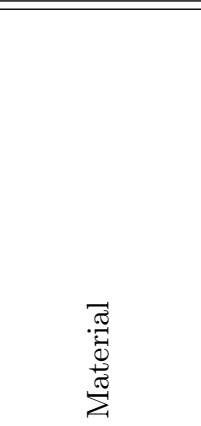 & 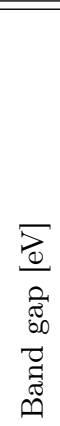 & 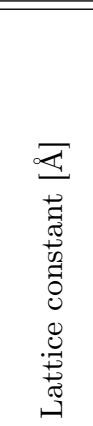 & 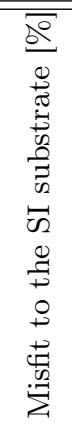 & 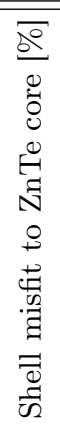 & 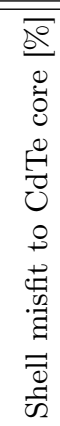 \\
\hline $\mathrm{Si}$ & 1.1 & 5.427 & 0 & - & - \\
\hline $\mathrm{ZnTe}$ & 2.3 & 6.103 & 12.5 & 0 & 5.9 \\
\hline $\mathrm{CdTe}$ & 1.5 & 6.483 & 19.5 & 6.2 & 0 \\
\hline $\mathrm{Cd}_{0.5} \mathrm{Zn}_{0.5} \mathrm{Te}$ & 1.9 & 6.293 & 16 & 3.0 & 3.0 \\
\hline
\end{tabular}

\section{Growth and structural investigation}

The II-VI NWs were grown on Si (111) substrate using similar procedure to Ref. [9]. Firstly, the Si substrate was inserted into the first MBE chamber, where $1 \mathrm{~nm}$ gold layer deposition took place. Then, the Si substrate was deoxidized in 50\% $\mathrm{HF}$ solution for $60 \mathrm{~s}$ and rinsed in the water, before inserting to the second, main MBE chamber. The substrate with the Au layer was heated up to $450{ }^{\circ} \mathrm{C}$, above the $\mathrm{Au} / \mathrm{Si}$ eutectic temperature, which induced formation of $\mathrm{Au} / \mathrm{Si}$ liquid droplets - catalysts for NW growth.

In the case of the $\mathrm{ZnTe} \mathrm{NWs}$, the $\mathrm{Zn}$ and Te (vapor) fluxes in the MBE chamber were used. We examined 2 types of the NWs, which slightly differ from each other. The main difference between them was the $\mathrm{Zn} / \mathrm{Te}$ flux ratio - 0.8 (for the first ones $-\mathrm{A}$ ) and 0.3 (for the second ones - B). The NWs A were grown at $360^{\circ} \mathrm{C}$ for $25 \mathrm{~min}$ with the $\mathrm{Zn}$ and Te fluxes: $8.0 \times 10^{-7}$ and $1.0 \times 10^{-6}$ mbar giving on average $0.7 \mu \mathrm{m} \mathrm{NWs}$ (Fig. 1a), whereas the NWs B were grown at $360^{\circ} \mathrm{C}$ for $83 \mathrm{~min}$ with the $\mathrm{Zn}$ and Te fluxes given by the partial pressures: $2.5 \times 10^{-7}$ and $9.8 \times 10^{-7}$ mbar, respectively with the length up to $2.5 \mu \mathrm{m}$ (Fig. 1b).

In the case of the CdTe NWs growth (Fig. 1c), it needs an initialization process by using $4 \mathrm{~min}$ deposition of the $\mathrm{ZnTe}$ in $380^{\circ} \mathrm{C}$ firstly [9], due to the high mismatch between the CdTe and the Si substrate (Table I). After the temperature reduction to $350^{\circ} \mathrm{C}$ the $\mathrm{CdTe}$ deposition takes place. The $\mathrm{Cd}$ and Te effusion cells were opened for $40 \mathrm{~min}$ which resulted in $1.5 \mu \mathrm{m}$ average length and $40 \mathrm{~nm}$ average diameter.

Furthermore, we examined the $\mathrm{ZnTe} /(\mathrm{Cd}, \mathrm{Zn}) \mathrm{Te}$ core/shell NWs (Fig. 1d). The ZnTe cores were axially grown in $380^{\circ} \mathrm{C}$ for $17 \mathrm{~min}$ in the same conditions as the ZnTe NWs (see above) according to the VLS mechanism. Next, the $(\mathrm{Cd}, \mathrm{Zn}) \mathrm{Te}$ part was performed for $7 \mathrm{~min}$ after lowering the temperature by about $40^{\circ} \mathrm{C}$, which was necessary for the incorporation $\mathrm{Cd}$ into ZnTe structure.

It is worth mentioning that the temperatures of the growth in high-vacuum MBE process are difficult to control and are quite approximated, especially in the case of NWs which are heated mainly by the radiation mechanism. Therefore, the temperature reduction involves the risk of freezing $\mathrm{Au} / \mathrm{Si}$ catalyst and stopping the VLS growth mechanism.

The crystal structure, the defects and the elemental composition characterization were performed using an imaging corrected Titan Cubed 80-300 microscope operating at $300 \mathrm{kV}$ and a double corrected JEM ARM operating at $200 \mathrm{kV}$ equipped with a windowless detector (X-MaxN 100 TLE from Oxford Instruments). The NWs were transferred from the Si substrate to the holey carbon copper grid by touching the surfaces together. A ZnTe NWs specimen was additionally prepared by focused ion beam (FIB) to observe the interface between the Si substrate and the epitaxial layer deposited during the nucleation and growth of the NWs. The high resolution transmission microscopy (HRTEM) and the annular dark 
field in the scanning transmission electron microscopy (STEM) mode were the main imaging techniques used in this work. The geometric phase analysis (GPA) algorithm [14] was used to measure the lattice strains in the electron microscopy images. Additionally, energy dispersive X-ray spectroscopy (EDX) provided the compositional insights of the NWs.

\section{1. $Z n T e N W s$}

The ZnTe NWs are a base for this work, as they are single cores grown directly on the Si (111) substrates. It is expected that, in the case of the 111-oriented surface, the ZnTe NWs will grow mainly vertically as the ones grown on 111-GaAs [11]. The randomly oriented ZnTe NWs were grown on the $\mathrm{Si}(111)$ as can be observed in the scanning electron microscopy (SEM) images in Fig. 1a,b. The key difference in the growth direction may lay in the (very) initial state of the NWs growth, which has not been well studied and understood so far. Therefore, we put forward a hypothesis based on SEM and TEM observations.

We presume that, before the NWs proper VLS growth, the ZnTe forms 3D islands directly on the Si substrate. They initially grow between the liquid catalyst droplets and the part of them is in epitaxial relation to the substrate. The $\mathrm{ZnTe}$ island might reach the droplet with $\{111\}$ facet, which gives opportunity to the NW growth on the contact surface of the ZnTe island, "under" the $\mathrm{Au}$ droplet starting the VLS mechanism. Basing on this assumption, the NWs might adopt to the $\{111\}$ island random orientations thus they seem not to be ordered (Fig. 1), contrary to the ordered growth on the GaAs substrate.

To investigate the $\mathrm{ZnTe} / \mathrm{Si}$ interface and the possible formation of the dislocations in the interfacial plane due to the high lattice mismatch, the cross-section FIB specimen was prepared. Figure 2 corresponds to the interface between the $\mathrm{Si}(111)$ and the epitaxial ZnTe island deposited on the substrate during MBE process. The HRTEM image (Fig. 2a) was taken along the [11-2] zone axis, therefore stacking faults and twin planes in the NWs were not revealed. Figure $2 \mathrm{~b}$ and $\mathrm{d}$ is the magnified views from Fig. 2a with the ball model crystal projections of the $\mathrm{Si}$ and $\mathrm{ZnTe}$, respectively. The fast Fourier transform (FFT) in Fig. 2c confirms the epitaxial relationship between the ZnTe island and the Si substrate. Figure $2 \mathrm{f}$ shows an atomically resolved magnified area (the black rectangle in Fig. 2a) taken at the interface between both materials. The Burgers vector circuits were drawn to determine the dislocation character of the defects observed at the interface. All these dislocations are $60^{\circ}$ type with 1/2 [1-10] Burgers vectors lying in the (111) plane and projected onto the [11-2] direction. Chen and Wang proposed two different misfit dislocation network models on the interface between c-BN single crystal and (111) diamond $(\approx 1.4 \%$ misfit). In their work [15]: (i) the misfit edge dislocation network with the hexagonal units and (ii) the partial misfit dislocations connected by the stacking faults. In our case, the growth of the ZnTe islands on the (111) Si substrate also form the interface between two fcc cubic materials along the [111] direction, although the mismatch is almost 10 times larger than the case described in Ref. [15]. Our data indicates to have the first misfit dislocation network model (the misfit edge dislocation network with the hexagonal units). The dislocation network between the ZnTe island and the $\mathrm{Si}(111)$ can be described along the $[11-2]$ projection by the additional Si plane in $1.7 \mathrm{~nm}$. To our knowledge, the examination of such an interface has never been reported yet.

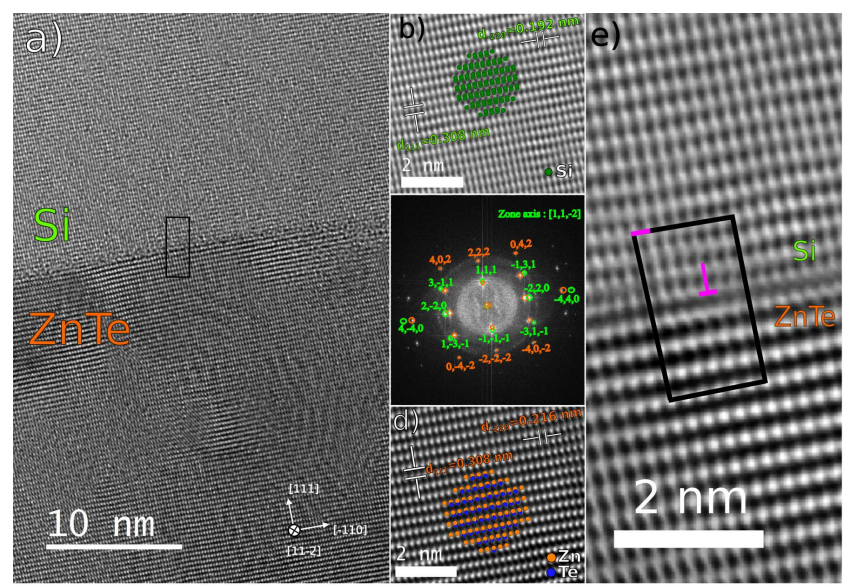

Fig. 2. (a) BF HRTEM image of the (111)Si and the ZnTe NW interface in the [11-2] zone axis with the fitted unit cells to the magnified regions (b) for the $\mathrm{Si}$ and (d) for the ZnTe. (c) Fast Fourier transform (FFT) of (a) with overlaid diffraction patterns of the Si (green) and the ZnTe (orange). (e) Magnified marked area from (a) with black rectangle encompassing magenta dislocations. Projected Burgers vector of $1 / 2$ [110] length is marked in magenta.

\section{2. $Z n T e N W s-A(Z n /$ Te ratio $=0.8)$}

The TEM images revealed two different NWs population. It mainly revealed the pure $\mathrm{ZB}$ phase in the NWs with the diameter ranging from 8 to $30 \mathrm{~nm}$ and several WZ NWs (approximately 20\%) with no stacking faults or other defects and the diameters from 2.5 to $13 \mathrm{~nm}$. The multiple twinning was observed in ZB NWs with very dense twinning planes, appearing even every 2 lattice planes in the [111] direction (Fig. 3d). The bright field TEM image in Fig. 3a corresponds to three NWs lying next to each other with the visible stacking faults perpendicular to the growth direction. We estimated the defect density by measuring the number of the SFs and the twinning boundaries per unit length $(\mu \mathrm{m})$ for each NWs (Fig. 3b). The fast Fourier transform from the yellow area in Fig. 3b is presented in Fig. 3c where the streak-like reflections are the evidence for the twinning boundaries or the SFs. Higher magnification image recorded in the NW with highest linear density of defects is shown in Fig. 3d. In general, we estimated to have in 
average $-770 \pm 200$ SFs and twinning boundaries together per $1 \mu \mathrm{m}$ in $\mathrm{ZB}$ structure (the number was ranging from 270 to 1300 ).

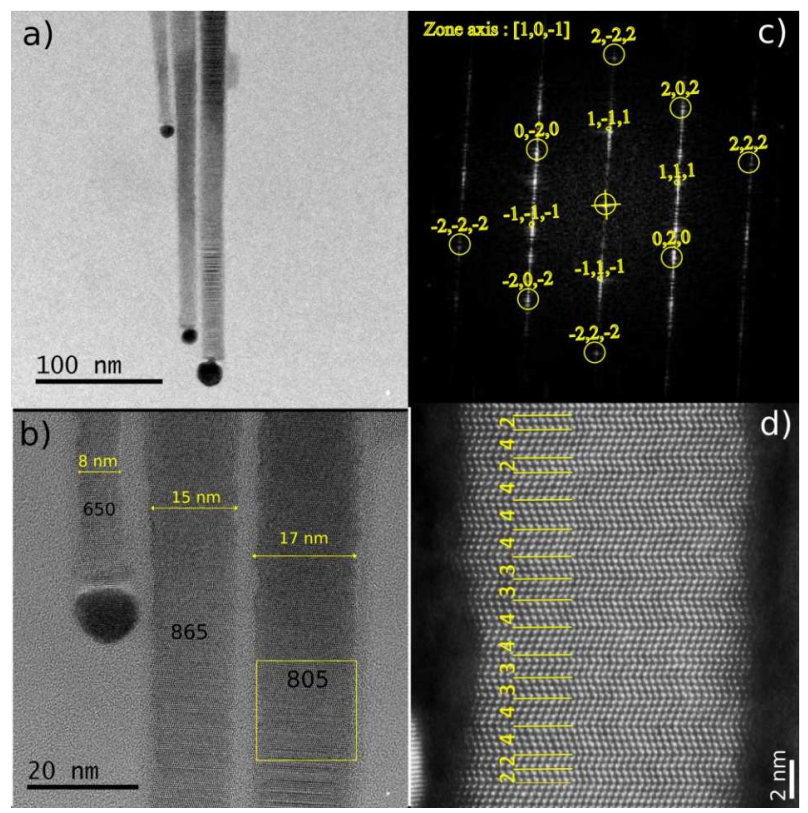

Fig. 3. (a) BF TEM image of 3 ZB NWs, (b) magnification of (a) with numbers of twinning boundaries per $1 \mu \mathrm{m}$ for each NW, (c) FFT of squared area from (b), (d) HAADF STEM of the middle NW from $(a, b)$ with marked twinning boundaries.

Figure 4a corresponds to the NW with pure $\mathrm{WZ}$ phase, confirmed by the FFT inset in Fig. 4b. The diameter of the WZ NWs was smaller than ZB ones (below $5 \mathrm{~nm}$ in the case of Fig. 4a and b). These results point out to an influence of the NW diameter on the ZnTe NW crystal structure. Interestingly, Fig. 4c corresponds to a NW with variable diameter $13-19 \mathrm{~nm}$. The image and FFT revealed that the structure changes from partially WZ structure (small diameter) to ZB twins superlattice (lager diameter), corroborating the transitions to the $\mathrm{ZB}$ structure by increasing the NW diameter.

$$
\text { 2.3. } Z n T e N W s-B(Z n / \text { Te ratio }=0.3)
$$

In the second sample - B, the ZnTe NWs diameter was noticeably larger than sample A, ranging from $28 \mathrm{~nm}$ to $50 \mathrm{~nm}$, having ZB structure (Fig. 5). Additionally, the ZnTe NWs - B are longer (up to $2.5 \mu \mathrm{m}$ ) than the ZnTe NWs - A due to the longer growth conditions $83 \mathrm{~min}$ in B vs. 25 min in A. A closer examination of the $\mathrm{ZnTe}$ NW-B revealed a very small amount of twinned bilayers (even none in some NWs) in comparison with A. Figure $5 \mathrm{~b}$ and d corresponds to two different NWs demonstrating the low or inexistent presence of twinning/stacking faults in this growth. The linear density measurements evidenced over 100 times less twinning boundaries/SF per $1 \mu \mathrm{m}$ than in the sample A (a maximum of $70 \mathrm{SF}$ per $1 \mu \mathrm{m}$ was estimated in this sample). Therefore, there is a remarkable effect of the II/VI ratio on the crystal

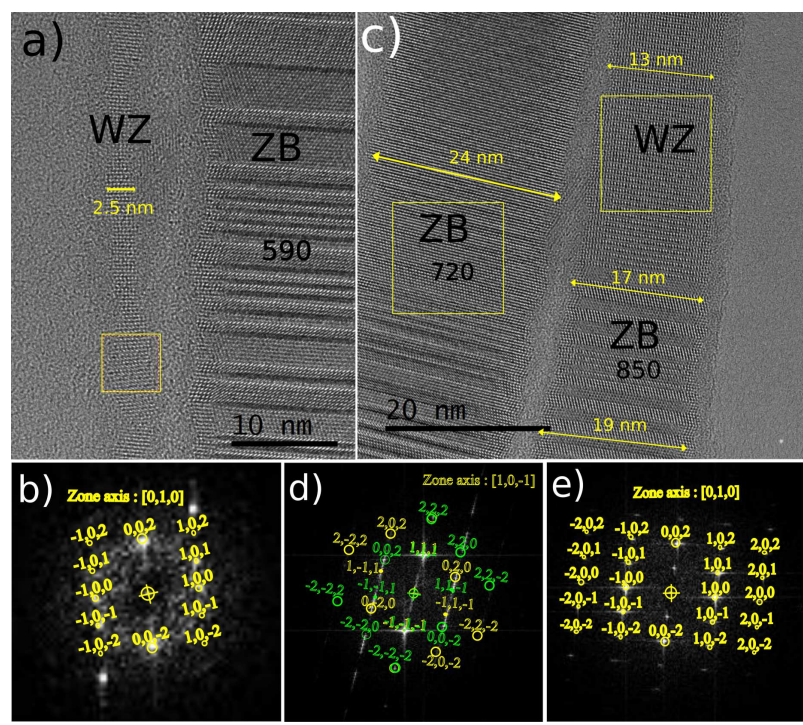

Fig. 4. BF TEM images with: (a) a part of ZB NW and a very thin wurtzite $(2.5 \mathrm{~nm}$ in diameter) NW with (b) FFT from yellow square with fitted ZnTe WZ structure, (c) a part of ZB NW with (d) FFT with fitted both ZB twins and (c) another $4.5 \mathrm{~nm}$ WZ NW with (e) diffraction pattern fitted to the FFT from marked area. Numbers on ZB NWs indicate densities of twinning boundaries per $1 \mu \mathrm{m}$.

structure in the case of the structure. A low $\mathrm{Zn} / \mathrm{Te}$ ratio promotes a $\mathrm{ZB}$ phase with very low twinning defects density. An increased in the $\mathrm{Zn} / \mathrm{Te}$ flux ratio promotes twinning and the transition to the $\mathrm{WZ}$ phase.

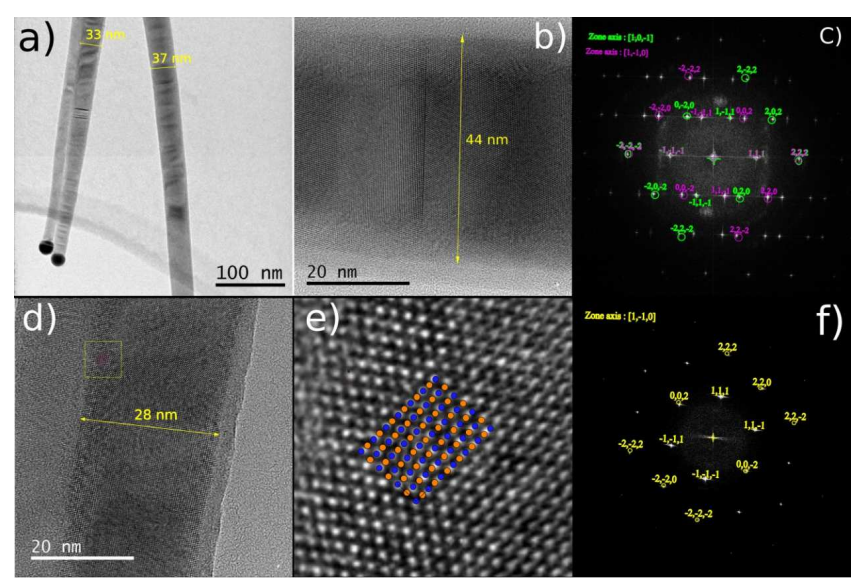

Fig. 5. Bright field TEM images of: (a) 3 NWs of 33$37 \mathrm{~nm}$ diameters with several visible SF perpendicular to the growth direction, (b) $44 \mathrm{~nm}$ in diameter NW with only one twinning boundary and (c) FFT from the whole image and both twins diffraction pattern fitting, (d) $28 \mathrm{~nm}$ in diameter, ZB defect-free NW with (e) magnified squared part with ZnTe ball model on it, (f) FFT from (d) and fitted diffraction pattern.

\section{4. $C d T e N W s$}

In the case of the CdTe NWs, it is assumed that their growth starts on the top of the ZnTe precursor islands 
or the short fragments of the ZnTe NWs. TEM analysis showed two different types of the NWs in the same sample; (i) WZ nanowires with a $\approx 25 \mathrm{~nm}$ (Fig. 6a) and (ii) $\mathrm{ZB}$ nanowires with $\mathrm{a} \approx 50 \mathrm{~nm}$ (Fig. 6b). Similar, average NW length was measured in both populations $\approx 1.5 \mu \mathrm{m}$ (Fig. 6c). The oblique stacking faults in the [-111] direction were observed in the ZB NWs (Fig. 6b). The contrast variations visible in Fig. 6 are observed due to the surface morphology.
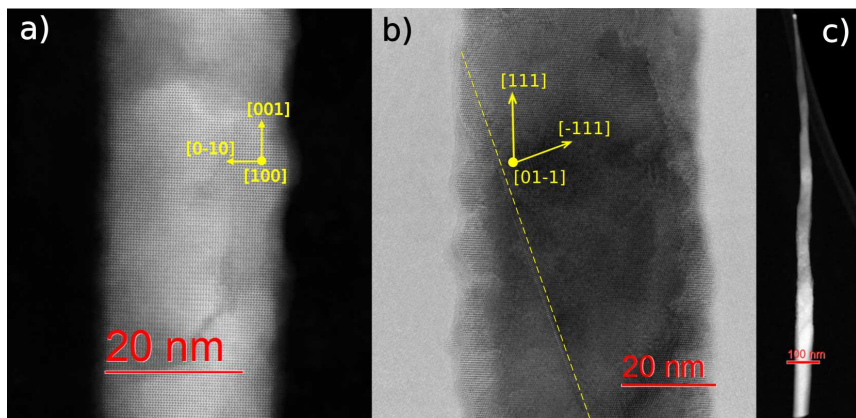

Fig. 6. (a) STEM image of the typical CdTe defectfree WZ NW, (b) BF TEM image of CdTe ZB NW with marked SF in oblique [-111] direction, (c) STEM image of CdTe ZB NW.

The another interesting observation is that whereas the ZB CdTe are defective the WZ CdTe was found to be defect-free (the SFs are not observed). Contrary to the ZB CdTe NWs, which are widely known, the WZ CdTe NWs are produced only by some methods [16-18] and the CdTe WZ NWs grown by MBE have not been reported yet. To our knowledge, only Wojnar et al. have reported the successful gold catalyzed WZ CdTe NWs growth by the VLS mechanism using the MBE process [5, 15].

Figure 7 shows the identification of both the ZB and the WZ NWs using selected area diffraction patterns and imaging. The magnified images, both the ZB (Fig. 7c) and the WZ (Fig. 7c) corresponds to the $\langle 110\rangle$ and the $\langle 11-20\rangle$ projections, respectively. A ball crystal model structure has been overlapped in the figure for the clarification. The high angle annular dark field (HAADF) high resolution STEM image recorded at the edge of the ZB NW (Fig. 6c) revealed the oblique stacking fault. The $\mathrm{Cd}-\mathrm{Te}$ dumbbell is also resolved in HAADF-STEM images along the $\langle 110\rangle$ projection. The analysis of the dumbbell intensity confirmed that the surface had the Te polarity - as previously reported in the ZnTe NW [19].

These results clearly illustrate that the WZ NWs as the diameter increases make a transition from the WZ to the $\mathrm{ZB}$ phase. This is in an agreement with the previous observations such as Ref. [15] when the diameter of NWs is relatively small. The NW diameters dispersion in the same sample can be explained by the differences in the Au catalytic particles, whose sizes are hard to control.

\section{5. $\mathrm{ZnTe} /(\mathrm{Cd}, \mathrm{Zn}) \mathrm{Te} N W \mathrm{~s}$}

The $\mathrm{ZnTe} /(\mathrm{Cd}, \mathrm{Zn}) \mathrm{Te}$ hetero-nanowires grown in coreshell geometry were analyzed by TEM. The ZnTe core
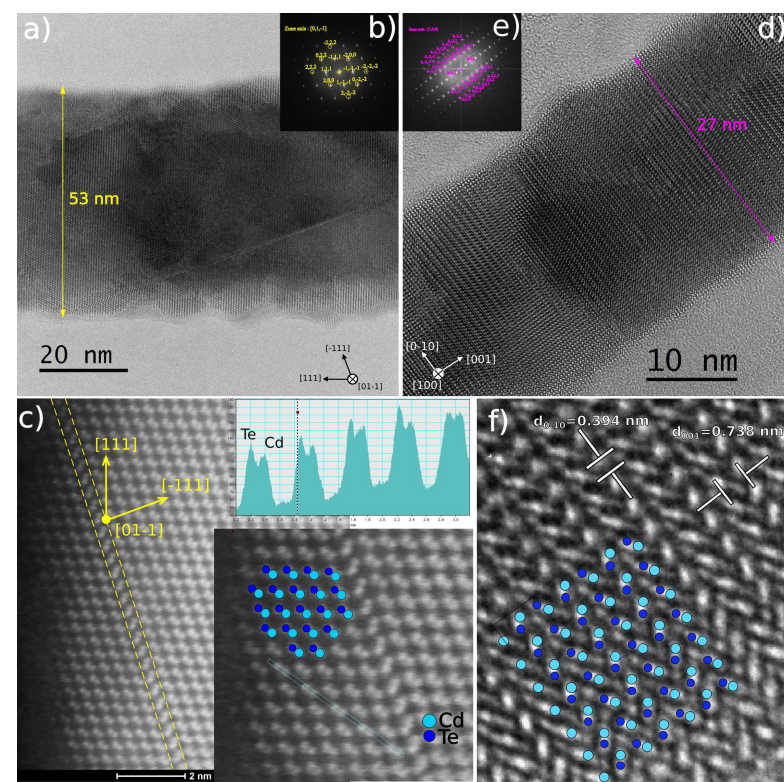

Fig. 7. (a) Bright field TEM image of $53 \mathrm{~nm}$ in diameter ZB NW with one stacking fault in [1-11] direction, (b) its FFT with fitted diffraction pattern in [1-10] zone axis, (c) NW from (a) with marked stacking fault and several unit cells overlaid on the structure, image taken in high resolution STEM enabling $\mathrm{Cd}-\mathrm{Te}$ dumbbell separation by their intensities, (d) BF TEM image of defect-free WZ NW of $27 \mathrm{~nm}$ average diameter with (e) FFT and fitted diffraction pattern in [100] zone axis, (f) filtered part of (d) with several unit cells overlaid on the structure.

was grown by the VLS mechanism while the $(\mathrm{Cd}, \mathrm{Zn}) \mathrm{Te}$ was grown on the side walls of the NW by the vaporsolid VS mechanism. The EDX line profile (Fig. 8) and the elemental mapping (Fig. 9) revealed the asymmetry in the thickness of the shell, which confirmed that the axial growth of the shell was not achieved, but the heterogeneous radial growth around the core occurred. The embedding of the core with the shell often results in the strong asymmetry in the thickness of the shell due to the geometric conditions of the MBE process (directional elemental fluxes, shadowing effect) which also impact on the strain distribution [8-10]. Experimental measurements showed a NW of $\approx 50 \mathrm{~nm}$, with a $\mathrm{ZnTe}$ core diameter of $20 \mathrm{~nm}$ and the thickness of the $(\mathrm{Cd}, \mathrm{Zn}) \mathrm{Te}$ shell changes from 10 to $20 \mathrm{~nm}$.

The HR-STEM investigations showed an epitaxial growth of the $(\mathrm{Cd}, \mathrm{Zn}) \mathrm{Te}$ shell on the ZnTe core. No misfit dislocation between the core and the shell were observed in the system (Fig. 10a,b) - the shell structure is exactly the same as the core structure with the SFs and the twin boundaries propagating from the core to the shell without any discontinuity. Therefore, the shell must be in compression in the axial and radial direction, due to the difference in the lattice constant.

Due to the radial difference of the thickness of the $(\mathrm{Cd}, \mathrm{Zn}) \mathrm{Te}$ shell, the NW axis is bended and the strain distribution becomes inhomogeneous and complex in 

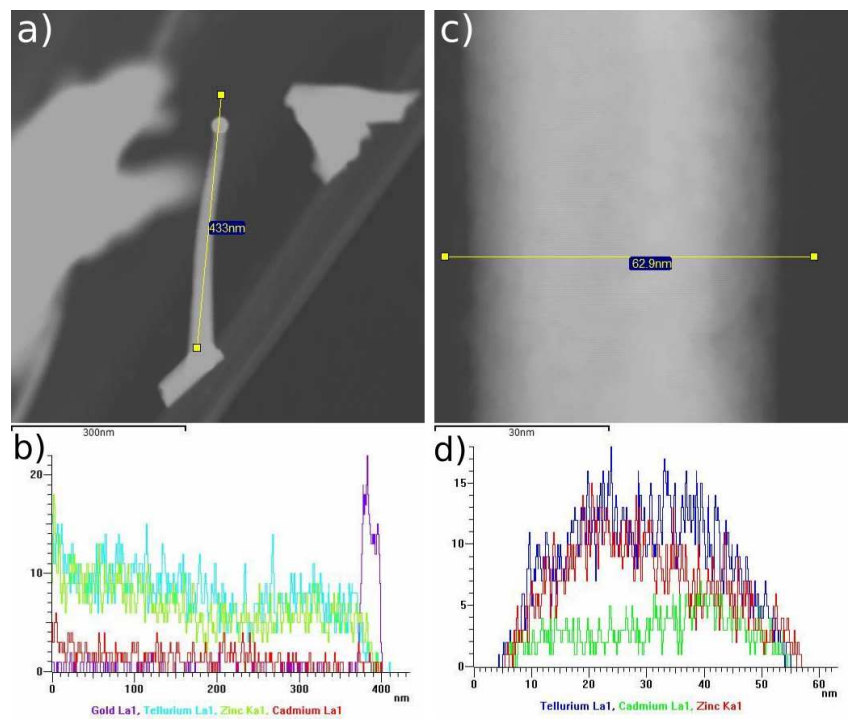

Fig. 8. (a) STEM image of a ZnTe/ZnCdTe NW with marked yellow line indicating (b) EDX integrated elements intensity profile spectrum along the NW, beginning from the bottom, (c) STEM image of a ZnTe/ZnCdTe NW with marked yellow line indicating (d) EDX integrated elements intensity profiles across the NW.
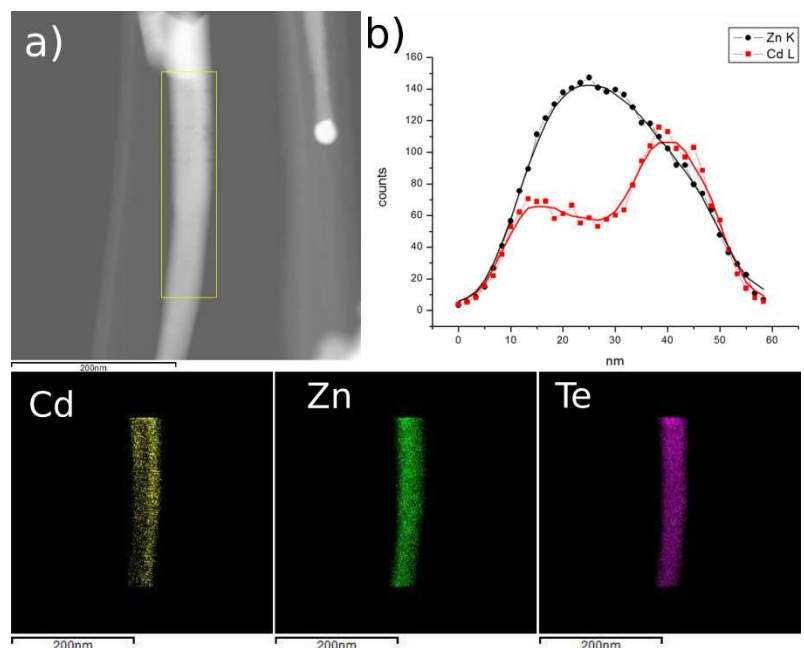

Te

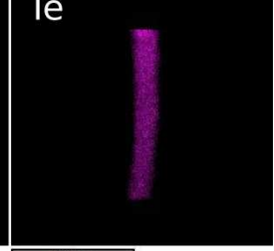

Fig. 9. (a) STEM image of a part of $\mathrm{ZnTe} / \mathrm{ZnCdTe}$ NW with marked yellow rectangle the area from which EDX intensity map was taken, regarding Cd (yellow), Zn (green), Te (magenta), (b) averaged amount of counts integrated across the NW in terms of $\mathrm{Zn} K$ and Cd $L$.

such a NW. The strain field was determined by analysing the projected strain tensor distribution using GPA method [19] of the HR-STEM image (Fig. 10a). Figure 10c and e corresponds to two components of the lattice distortion tensor projected along $\langle 110\rangle$ zone axis: radial $\varepsilon_{R}$ and axial $\varepsilon_{Z}$, respectively. The position of the ZnTe core and the asymmetry of the $(\mathrm{Cd}, \mathrm{Zn}) \mathrm{Te}$ shell are clearly visible (red parts in the black rectangle). The averaged radial $\varepsilon_{R}$ value along the axial direction is presented in
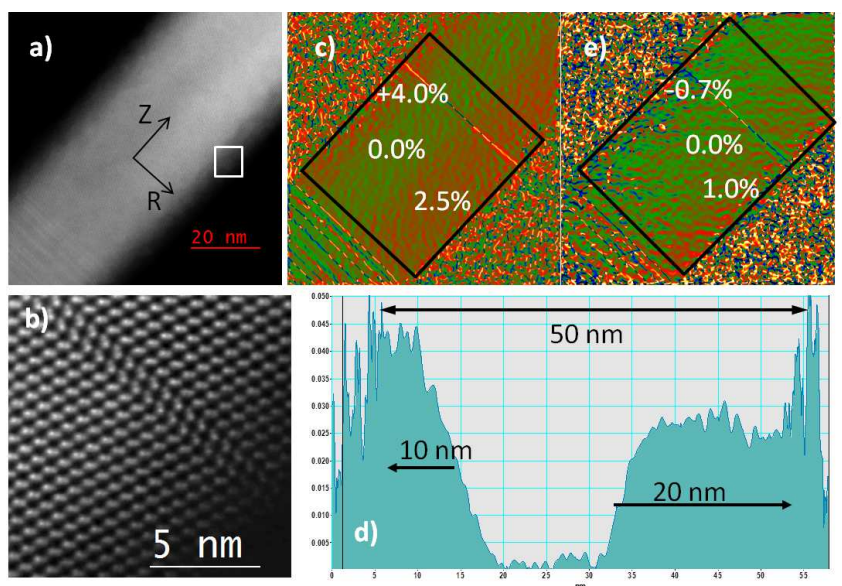

Fig. 10. (a) HR-STEM image of $\mathrm{ZnTe} / \mathrm{ZnCdTe} \mathrm{NW}$; (b) magnification of (a) with $\mathrm{SF} /$ twinning planes perpendicular to the growth direction from white rectangle; (c) the projected radial component $\varepsilon_{R}$ of strain tensor obtained by GPA of image (a); (d) relative value of $\varepsilon_{R}$ across the NW with the average signal from black rectangle in $(\mathrm{c}) ;(\mathrm{e})$ relative value of $\varepsilon_{Z}$ component of projected lattice distortion tensor.

Fig. 10d - the $\varepsilon_{R}$ was rescaled to be zero in the area corresponding to the ZnTe core. Since $\varepsilon_{R}$ is proportional to the lattice constant, Fig. 10d suggests that concentration of cadmium is higher in thinner shell (left part higher intensity) than in thicker part (right part) which agrees with the EDX elemental distribution.

However, due to the bending of the NW, the thinner shell is under high tensile strain, which results in the radial expansion of the lattice constant. In Fig. 10e the $2 \mathrm{D}$ map of $\varepsilon_{Z}$ component is present. The lattice deformation in the core constitutes a $0.0 \%$ reference to other parts of the shell. It is visible that the thinner shell is compressed to $-0.7 \%$ when the thicker part is stretched to $+1.0 \%$. This fact can explain the observed asymmetry in $\varepsilon_{R}$ component.

\section{Conclusions}

We confirm that the interface between the $\mathrm{Si}(111)$ substrate and the $\mathrm{ZnTe}(111)$ island have the misfit dislocation network, consisting of the hexagonal units with full edge dislocations model [15]. Then, we measured two types of the ZnTe NWs with different Zn to Te fluxes ratio. It turned out that the NWs from A sample having $0.8 \mathrm{Zn} /$ Te ratio ( $\mathrm{Zn}$ and Te fluxes: $8.0 \times 10^{-7}$ and $1.0 \times 10^{-6}$ mbar) grew in two phases: mainly in the ZB structure with the superlattice of twins with very dense twinning boundaries appearing even every two lattice planes in the [111] direction of the diameters ranging from 8 to $30 \mathrm{~nm}$ and rarely in the WZ structure free of defects of the diameters ranging from 2.5 to $13 \mathrm{~nm}$. Whereas, B sample of the $\mathrm{ZnTe} N W$ s having $0.3 \mathrm{Zn} / \mathrm{Te}$ ratio $\left(2.5 \times 10^{-7}\right.$ and $\left.9.8 \times 10^{-7} \mathrm{mbar}\right)$, formed almost defectfree ZB structure (with several SFs perpendicular to the growth direction). Definitely, the larger $\mathrm{Zn} / \mathrm{Te}$ fluxes ra- 
tio is, the more SFs are in the structure of such grown NWs.

In the case of CdTe NWs, we also observed NWs of two different structures: the ZB NWs with $50 \mathrm{~nm}$ average diameter and defect-free WZ NWs (never obtained by the MBE, supported by the VLS mechanism yet) with the diameter around $25 \mathrm{~nm}$. The ZB NWs were characterized by oblique $\mathrm{SF}$ in the [-111] direction. We presume that the appearance of the thin WZ NWs might be caused by the uncontrolled process of $\mathrm{Au}$ gold droplets formation, so that smaller droplets can be the catalysts for thinner $\mathrm{NW}$, as they form the structure on $\mathrm{Au} / \mathrm{NW}$ interface.

The EDX and GPA analysis of the ZnTe/(Cd,Zn)Te NWs show that the growth of the core-shell NWs by the MBE process depends strongly on the geometry. The orientation of the NW in relation to the atomic fluxes in the MBE is critical. The angular inhomogeneous thickness of the shell can bend NWs and can introduce the heterogeneous strain field which can have impact on the optical properties of such hetero-nano-objects.

In this stage of the research, we are not able to connect the exact crystallographic structure of the NW with its cathodoluminescence in the SEM. The luminescence signal is averaged for the whole sample. We are planning to enhance the method and examine the same NWs in SEM and TEM.

\section{Acknowledgments}

The research was partially supported by National Science Centre (Poland) by grant No. DEC-2011/01/D/ST5/05039 and DEC2016/21/B/ST5/03411. TEM investigation was supported by European Regional Development Fund through the Innovative Economy grant No. POIG.02.0100-14-032/08 and EU 7th Framework Programme under the CAPACITIES project REGPOT-CT-2013-316014 (EAgLE) co-financed by Polish Ministry of Science and Higher Education, Grant Agreement 2819/7.PR/2013/2.

\section{References}

[1] C. Thelander, T. Mårtensson, M.T. Björk, B.J. Ohlsson, M.W. Larsson, L.R. Wallenberg, L. Samuelson, Appl. Phys. Lett. 83, 2052 (2003).

[2] S. Thunich, L. Prechtel, D. Spirkoska, G. Abstreiter, A.F.I. Morral, A.W. Holleitner, Appl. Phys. Lett. 95, 083111 (2009).

[3] F. Qian, Y. Li, S. Gradecak, H.G. Park, Y.J. Dong, Y. Ding, Z.L. Wang, C.M. Lieber, Nature Mater. 7, 701 (2008).

[4] B. Tian, X. Zheng, T.J. Kempa, Y. Fang, N. Yu, G. Yu, J. Huang, C.M. Lieber, Nature 449, 885 (2007).
[5] T. Wojtowicz, E. Janik, W. Zaleszczyk, J. Sadowski, G. Karczewski, P. Dłużewski, S. Kret, W. Szuszkiewicz, E. Dynowska, J. Domagała, M. Aleszkiewicz, L.T. Baczewski, A. Petroutchik, J. Kor. Phys. Soc. 53, 3055 (2008).

[6] E. Janik, J. Sadowski, P. Dluzewski, S. Kret, L.T. Baczewski, A. Petroutchik, E. Lusakowska, J. Wrobel, W. Zaleszczyk, G. Karczewski, T. Wojtowicz, A. Presz, Appl. Phys. Lett. 89, 133114 (2006).

[7] E. Janik, P. Dłużewski, S. Kret, A. Presz, H. Kirmse, W. Neumann, W. Zaleszczyk, L.T. Baczewski, Nanotechnology 18, 475606 (2007).

[8] P. Wojnar, W. Zaleszczyk, L. Klopotowski, E. Janik, M. Wiater, L.T. Baczewski, S. Kret, G. Karczewski, J. Kossut, T. Wojtowicz, Nanotechnology 24, 365201 (2013).

[9] P. Wojnar, J. Płachta, S. Kret, A. Kaleta, W. Zaleszczyk, M. Szymura, M. Wiater, L.T. Baczewski, A. Petruchik, G. Karczewski, J. Kossut, T. Wojtowicz, Nanotechnology 28, 112139 (2016).

[10] P. Wojnar, E. Janik, L.T. Baczewski, S. Kret, E. Dynowska, T. Wojciechowski, J. Suffczyński, J. Papierska, P. Kossacki, G. Karczewski, J. Kossut, T. Wojtowicz, Nano Lett. 12, 3404 (2012).

[11] R.S. Wagner, W.C. Ellis, Appl. Phys. Lett. 4, 89 (1964).

[12] P. Wojnar, E. Janik, L.T. Baczewski, S. Kret, G. Karczewski, T. Wojtowicz, M. Goryca, T. Kazimierczuk, P. Kossacki, Appl. Phys. Lett. 99, 113109 (2011).

[13] P. Wojnar, J. Płachta, W. Zaleszczyk, S. Kret, A.M. Sanchez, R. Rudniewski, K. Raczkowska, M. Szymura, G. Karczewski, L. Baczewski, A. Pietruczik, T. Wojtowicz, J. Kossut, Nanoscale 8, 5720 (2016).

[14] S. Kret, P. Ruterana, A. Rosenauer, D. Gerthsen, Phys. Status Solidi B 227, 247 (2001).

[15] C. Chen, Z. Wang, T. Kato, N. Shibata, T. Taniguchi, Y. Ikuhara, Nature Commun. 6, 6327 (2015).

[16] S. Neretina, R.A. Hughes, J.F. Britten, N.V. Sochinskii, J.S. Preston, P. Mascher, Nanotechnology 18, 275301 (2007).

[17] B. Luo, Y. Wang, M. Tan, L. Cao, Wei Zhu, Cryst. Eng. Comm. 14, 7922 (2012).

[18] X. Wang, J. Wang, M. Zhou, H. Zhu, H. Wang, X. Cui, X. Xiao, Q. Li, J. Phys. Chem. C 113, 16951 (2009).

[19] M. de la Mata, C. Magen, J. Gazquez, M.I. Bakti Utama, M.N. Heiss, S. Lopatin, F. Furtmayr, C.J. Fernández-Rojas, B. Peng, J.R. Morante, R. Rurali, M. Eickhoff, A. Fontcuberta i Morral, Q. Xiong, J. Arbiol, Nano Lett. 12, 2579 (2012). 